\title{
Evidence of Selection Against Damaged Mitochondria During Early Embryogenesis in the Mouse
}

\author{
Thiago S. Machado 1,2, Carolina H. Macabelli', Maite Del Collado ${ }^{3}$, Flávio V. Meirelles ${ }^{2,3}$, \\ Francisco E. G. Guimarães ${ }^{4}$ and Marcos R. Chiaratti ${ }^{1,2 *}$

\begin{abstract}
' Departamento de Genética e Evolução, Universidade Federal de São Carlos, São Carlos, Brazil, ${ }^{2}$ Faculdade de Medicina Veterinária e Zootecnia, Universidade de São Paulo, São Paulo, Brazil, ${ }^{3}$ Faculdade de Zootecnia e Engenharia de Alimentos, Universidade de São Paulo, Pirassununga, Brazil, ${ }^{4}$ Instituto de Física de São Carlos, Universidade de São Paulo, São Carlos, Brazil
\end{abstract}

\section{OPEN ACCESS}

Edited by:

Aurora Gomez-Duran, University of Cambridge, United Kingdom

Reviewed by: Justin St. John

The University of Adelaide, Australia Zhongxiong Lai,

Fujian Agriculture and Forestry University, China

*Correspondence: Marcos R. Chiaratti marcos.chiaratti@ufscar.br

Specialty section: This article was submitted to Epigenomics and Epigenetics, a section of the journal

Frontiers in Genetics

Received: 07 September 2019 Accepted: 29 June 2020 Published: 15 July 2020

Citation:

Machado TS, Macabelli $\mathrm{CH}$, Collado MD, Meirelles FV Guimarães FEG and Chiaratti MR (2020) Evidence of Selection Against Damaged Mitochondria During Early

Embryogenesis in the Mouse.

Front. Genet. 11:762.

doi: 10.3389/fgene.2020.00762
There is evidence of a purifying filter acting in the female germline to prevent the expansion of deleterious mutations in the mitochondrial DNA (mtDNA). Given our poor understanding of this filter, here we investigate the competence of the mouse embryo to eliminate dysfunctional mitochondria. Toward that, mitochondria were damaged by photoirradiation of NZB/BINJ zygotes loaded with chloromethyl-X-rosamine (CMXRos). The resultant cytoplasm was then injected into C57BL/6J zygotes to track the levels of NZB/BINJ mtDNA during the preimplantation development. About $30 \%$ of NZB/BINJ mtDNA was present after injection, regardless of using photoirradiated or non-photoirradiated cytoplasmic donors. Moreover, injection of photoirradiatedderived cytoplasm did not impact development into blastocysts. However, lower levels of NZB/BINJ mtDNA were present in blastocysts when comparing injection of photoirradiated $(24.7 \% \pm 1.43)$ versus non-photoirradiated $(31.4 \% \pm 1.43)$ cytoplasm. Given that total mtDNA content remained stable between stages (zygotes vs. blastocysts) and treatments (photoirradiated vs. non-photoirradiated), these results indicate that the photoirradiated-derived mtDNA was replaced by recipient mtDNA in blastocysts. Unexpectedly, treatment with rapamycin prevented the drop in NZB/BINJ mtDNA levels associated with injection of photoirradiated cytoplasm. Additionally, analysis of mitochondria-autophagosome colocalization provided no evidence that photoirradiated mitochondria were eliminated by autophagy. In conclusion, our findings give evidence that the mouse embryo is competent to mitigate the levels of damaged mitochondria, which might have implications to the transmission of mtDNAencoded disease.

Keywords: mitochondria, embryo, mitochondrial DNA, mtDNA, mouse, cytoplasmic transfer, NZB, photosensitization

\section{INTRODUCTION}

Mitochondria play a central role in cellular energy production (i.e., ATP), besides being involved in several other functions including $\mathrm{Ca}^{2+}$ buffering, innate immunity, biogenesis of iron-sulfur clusters and apoptosis (Wallace and Chalkia, 2013). Most proteins needed for mitochondrial function are encoded in the nucleus and imported by the organelle (Wallace and Chalkia, 2013). Yet, the mitochondrion also relies on 37 genes ( 13 mRNAs, 22 tRNAs, and two rRNAs) encoded by its own genome, the mitochondrial DNA (mtDNA). The importance of these genes is revealed 
by mutations in mtDNA, which can result in mitochondrial dysfunction and severe pathologies in humans (Stewart and Chinnery, 2015). Manifestation of these pathologies is difficult to predict though as it depends on the level of mutant mtDNA. Multiple copies of mtDNA are present in each cell and mutations commonly coexist with wild-type molecules, a condition termed heteroplasmy (Schon et al., 2012; Burr et al., 2018). Given that most mtDNA mutations are recessive, wild-type molecules can complement the mutation defect. A threshold level of mutant mtDNA is needed to impair mitochondrial function (i.e., 60$80 \%$ ), but this threshold level varies for different mutations and tissues (Schon et al., 2012; Burr et al., 2018).

Due to the lack of efficient methods to treat mitochondrial disease, much attention has been given to prevent its transmission to the next generation. Yet, the non-Mendelian pattern of mtDNA inheritance makes difficult to predict transmission of such disease (Stewart and Chinnery, 2015). Despite few exceptions (Luo et al., 2018), autophagic elimination of paternal mitochondria shortly after fertilization assures mtDNA to be exclusively inherited from the mother (Rojansky et al., 2016; Wei et al., 2020). In addition, in case of heteroplasmy, several mechanisms take place during germline development toward reestablishing homoplasmy (i.e., existence of a single mtDNA genotype, regardless of mutant or wild-type). For instance, the mitochondrial genetic bottleneck allows for quick changes in mtDNA genotype frequency (Jenuth et al., 1996; Cree et al., 2008; Wai et al., 2008; Floros et al., 2018; Latorre-Pellicer et al., 2019). Also, there is increasing evidence in support of purifying selection acting in the female germline to prevent deleterious mutations (i.e., non-synonymous) in mtDNA from accumulating in the population (Burr et al., 2018).

One of the most consistent evidence of purifying selection first came from the work by Stewart et al. (2008). Using a mouse model with a burden of randomly generated mtDNA mutations, the authors found that synonymous mutations in protein-coding genes are preferentially transmitted to offspring than non-synonymous mutations. In addition, mutations in tRNA and rRNA genes were more often present in offspring than mutations in protein-coding genes (Stewart et al., 2008). Similar findings have been reported in mice and humans (Sato et al., 2007; Fan et al., 2008; Freyer et al., 2012; Sharpley et al., 2012; Li et al., 2016; Floros et al., 2018; Latorre-Pellicer et al., 2019; Wei et al., 2019). However, the mechanism underpinning purifying selection is currently unclear (Burr et al., 2018). Here we provide evidence that early embryos mitigate the levels of photoirradiated mitochondria introduced by cytoplasmic transfer (CT), which suggests they are virtually competent to tackle dysfunctional mitochondria harboring deleterious mtDNA mutations.

\section{MATERIALS AND METHODS}

All chemical and reagents were purchased from Sigma-Aldrich Chemical Co. (St. Louis, MO, United States), unless otherwise stated. All experiments were performed in compliance with the regulations and policies of the National Council for Control of Animal Experimentation (CONCEA, Brazil) and were approved by the Animal Care and Use Committee at Universidade de São Paulo (USP_-protocol number 13.1.1832.74.8).

\section{Source of Mouse and Embryos}

Mice containing mtDNA of NZB/BINJ (NZB) origin were obtained by backcrossing NZB females to C57BL/6J (B6) females for five generations. Thereafter, females with NZB mtDNA in a $\sim 100 \%$ B6 background were maintained by brother-sister mating (Machado et al., 2015). Mice containing mtDNA of B6 origin were obtained from F1 females from a cross of B6 females with males of CBA origin. Mice with mtDNA of NZB or B6 origin are hereafter termed NZB and B6, respectively.

To obtain pronuclear zygotes, females were intraperitoneally injected with 5 I.U. of equine chorionic gonadotropin (eCG; Folligon, MSD Animal Health, Summit, United States) and 5 I.U. of human chorionic gonadotropin (hCG; Chorulon, MSD Animal Health), given 46-47 h apart. Immediately after the hCG injection, females were paired with B6 males and inspected for the presence of vaginal plug in the next morning. Pronuclear zygotes were collected from the oviduct (ampulla) of plugged females $\sim 18 \mathrm{~h}$ after the hCG injection using HEPES-buffered KSOM medium (Erbach et al., 1994; Nagy et al., 2003; Machado et al., 2015). Viable zygotes were denuded of cumulus cells by vigorous pipetting in the presence of $0.3 \%$ hyaluronidase in HEPES-buffered KSOM. Groups of 20 zygotes were cultured in vitro under mineral oil in a $40 \mu \mathrm{l}$ drop of KSOM. After $96 \mathrm{~h}$ of culture in an incubator (set at $37^{\circ} \mathrm{C}$, maximum humidity, and $5 \% \mathrm{CO}_{2}$ in air), embryos were assessed as for the blastocyst rate (Nagy et al., 2003; Machado et al., 2015).

\section{Induction of Mitochondrial Damage}

Chloromethyl-X-rosamine (CMXRos; MitoTracker Red; ThermoFisher Scientific, Waltham, MA, United States) is a mitochondrion-selective fluorescent probe with a strong photosensitizing action (Minamikawa et al., 1999; Lum et al., 2002; Palermo et al., 2002; Lum and Nagley, 2003; Takeuchi et al., 2005). Under photoirradiation, CMXRos absorbs light, leading to excitation of the outer shell electrons and generation of reactive species such as hydroxyl radicals and singlet oxygen within the mitochondrion. These reactive species may damage mitochondrial structures, with evidence of organelle swelling and membrane depolarization (Minamikawa et al., 1999; Lum et al., 2002; Palermo et al., 2002; Lum and Nagley, 2003; Takeuchi et al., 2005).

To induce mitochondrial damage, NZB zygotes at the pronuclear stage were incubated for $30 \mathrm{~min}$ with $500 \mathrm{nM}$ CMXRos in HEPES-buffered KSOM at $37^{\circ} \mathrm{C}$. Next, based on a previous report (Takeuchi et al., 2005), zygotes were rinsed three times in HEPES-buffered KSOM and photoirradiated for either $0,2.5,5,10,20$, or $60 \mathrm{~s}$. Photoirradiation was performed in groups of 20 zygotes using an inverted microscope (Eclipse TS 100, Nikon Instruments Inc., Tokyo, Japan) equipped with an epifluorescence attachment (50-W mercury burner) with a Texas Red filter (excitation wavelength, 540-580 nm; emission wavelength, 600-660 nm) at 200x magnification (Minamikawa et al., 1999; Lum et al., 2002; Palermo et al., 2002; Takeuchi et al., 2005). Control zygotes were photoirradiated for either 0 or $60 \mathrm{~s}$ without prior loading with CMXRos. 


\section{Cytoplasmic Transfer}

Five experimental groups were considered during CT experiments: control B6 embryos not subjected to either CMXRos loading or photoirradiation-termed "B6control"; NZB embryos subjected to CMXRos loading and photoirradiation (P) for either 0 or $20 \mathrm{~s}$-termed "NZB-P0" and "NZB-P20," respectively; and, B6 embryos subjected to CT using cytoplasm from either NZB-P0 or NZB-P20-termed "CT-P0" and "CT-P20", respectively.

Micromanipulation was performed using an inverted microscope (Leica DMI RB, Leica, Wetzlar, Germany) equipped with micromanipulators and microinjectors (Narishige, Tokyo, Japan), as previously reported (Machado et al., 2015). Briefly, pronuclear zygotes were incubated for $15 \mathrm{~min}$ in HEPES-buffered KSOM medium containing $5 \mu \mathrm{g} / \mathrm{ml}$ cytochalasin and $5 \mu \mathrm{g} / \mathrm{ml}$ nocodazole. Next, $\sim 30 \%$ of cytoplasm was removed from B6 zygotes (calculated as previously reported; Chiaratti et al., 2010), followed by injection in the perivitelline space of a similar amount of cytoplasm derived from NZB zygotes. Pronuclei were always visualized during the micromanipulation procedure to prevent their unintended removal. After micromanipulation, zygotes were placed in an electrofusion solution $(0.28 \mathrm{M}$ mannitol, $0.1 \mathrm{mM} \mathrm{MgSO}_{4}, 0.5 \mathrm{mM}$ HEPES, and $0.05 \% \mathrm{BSA}$ ) and subjected to a single electrical pulse of $1 \mathrm{kV} / \mathrm{cm}$ (DC) for $45 \mu \mathrm{s}$ (Multiporator, Eppendorf, Hamburg, Germany) to induce fusion of the NZB cytoplast with the B6 recipient zygote. Successfully fused zygotes were cultured in vitro as described above. When applicable, embryos were cultured in the presence of $250 \mathrm{nM}$ rapamycin (Lee et al., 2011; Gilkerson et al., 2012). After 96 h of in vitro culture, the blastocyst rate was assessed.

\section{Evaluation of NZB Levels and Mitochondrial DNA Copy Number}

Embryos used for molecular evaluation were sampled immediately before (at the pronuclear stage) or after (at the blastocyst stage) in vitro culture. These were rinsed three times in phosphate buffer solution (PBS) containing $0.1 \%$ polyvinyl pyrrolidone (PVP) and stored individually in $1 \mu \mathrm{l} \mathrm{PBS}$ plus $0.1 \%$ PVP in $0.2 \mathrm{ml}$ tubes at $-20^{\circ} \mathrm{C}$. Embryos were lyzed for $3 \mathrm{~h}$ at $55^{\circ} \mathrm{C}$ in $50 \mathrm{mM} \mathrm{KCl}, 10 \mathrm{mM}$ Trix- $\mathrm{Cl}$ (pH 8.3), $2 \mathrm{mM} \mathrm{MgCl} 2,0.1 \mathrm{mg} / \mathrm{ml}$ gelatin, $0.45 \%$ Igepal CA-630, 0.45\% Tween 20 , and $125 \mu \mathrm{g} / \mathrm{ml}$ proteinase $\mathrm{K}$ (ThermoFisher Scientific). Following, lysates were incubated at $95^{\circ} \mathrm{C}$ for $10 \mathrm{~min}$ for proteinase $\mathrm{K}$ inactivation, diluted with $45 \mu \mathrm{l}$ ultrapure $\mathrm{H}_{2} \mathrm{O}$, and centrifuged at 10,000 $\times g$ for $5 \mathrm{~min}$. The supernatant was finally used for analysis of NZB levels and mtDNA copy number (Machado et al., 2015).

The levels of NZB mtDNA in zygotes and blastocysts were assessed by quantitative PCR (qPCR) as previously reported by Machado et al. (2015). Briefly, two set of primers were used to amplify either a 118-bp fragment of NZB mtDNA or a 146-bp fragment of B6 mtDNA. Reactions consisted of a final volume of $15 \mu \mathrm{l}$ containing $5 \mu \mathrm{l}$ of sample lysate, $200 \mathrm{nM}$ of each primer, and 1x Power SYBR Green Master Mix (ThermoFisher Scientific). Amplifications were performed using the 7500 Fast Real-Time PCR System (ThermoFisher Scientific) and the following cycling conditions: $95^{\circ} \mathrm{C}$ for $10 \mathrm{~min}$, followed by 40 cycles of $95^{\circ} \mathrm{C}$ for
$15 \mathrm{~s}$, and $62^{\circ} \mathrm{C}$ for $1 \mathrm{~min}$. SYBR Green fluorescence was read at the end of each extension step. The percentage of NZB mtDNA was calculated in relation to the sum of NZB and B6 mtDNA, as reported by Machado et al. (2015).

Total mtDNA copy number (sum of NZB and B6 mtDNA) in zygotes and blastocysts was assessed as reported by Machado et al. (2015). Toward that aim, a 736-bp fragment of B6 mtDNA was cloned into a plasmid vector (pCR2.1-TopTA; ThermoFisher Scientific). Part of this construct (at concentration of $10^{7}, 10^{6}$, $10^{5}, 10^{4}$, and $10^{3}$ copies/reaction) was amplified by qPCR in parallel with zygote and blastocyst samples. Conditions of qPCR were the same described above, except for the use of nondiscriminative primers that amplify a common fragment (148 bp) from both NZB and B6 mtDNA. The number of mtDNA copies was calculated as reported by Machado et al. (2015).

\section{Analysis of \\ Mitochondria-Autophagosome Colocalization}

Embryos at the two-cell stage ( $21 \mathrm{~h}$ of culture) were fixed in 3.7\% paraformaldehyde in PBS with $0.5 \%$ Triton X-100 and 0.1\% PVP for $15 \mathrm{~min}$ at room temperature. Next, embryos were rinsed three times in PBS with $0.1 \%$ PVP, and incubated for $1 \mathrm{~h}$ at room temperature with a primary antibody (anti-MAP1LC3B raised in rabbit; Cat\# L7543, Sigma-Aldrich). Afterward, embryos were rinsed in PBS with 0.1\% PVP, and incubated for $1 \mathrm{~h}$ at room temperature with an Alexa Fluor 488-tagged secondary antibody raised against rabbit (Cat\# A11008, ThermoFisher Scientific). Both antibodies were diluted 1:200 in PBS with 0.1\% PVP. Finally, embryos were thoroughly washed in PBS with $0.1 \%$ PVP, and mounted on slides with coverslips using Prolong Gold (ThermoFisher Scientific). Embryos were evaluated by confocal microscopy (LSM 780, Zeiss, Oberkochen, Germany) at $1000 \mathrm{x}$ magnification. Autophagosomes were visualized at 495 and $519 \mathrm{~nm}$, respectively, for excitation and emission. NZB mitochondria (previously stained with CMXRos for the photosensitization treatment) were visualized at 543 and $580-650 \mathrm{~nm}$, respectively. Images were analyzed using the ZEN lite (Zeiss).

\section{Statistical Analyses}

Statistical analyses were performed using SAS v.9.3 (SAS/STAT, SAS Institute Inc., Cary, NC, United States). When necessary, data were transformed to fit a normal distribution. Data were analyzed by one-way or two-way ANOVA followed by Tukey's post hoc test. Values are reported as mean \pm standard error of the mean (SEM).

\section{RESULTS}

\section{Photoirradiation of CMXRos-Loaded Zygotes Prevents Development Into Blastocysts}

Aiming to set up photosensitization conditions, zygotes were loaded with CMXRos and photoirradiated for either 0, 2.5, 5, 


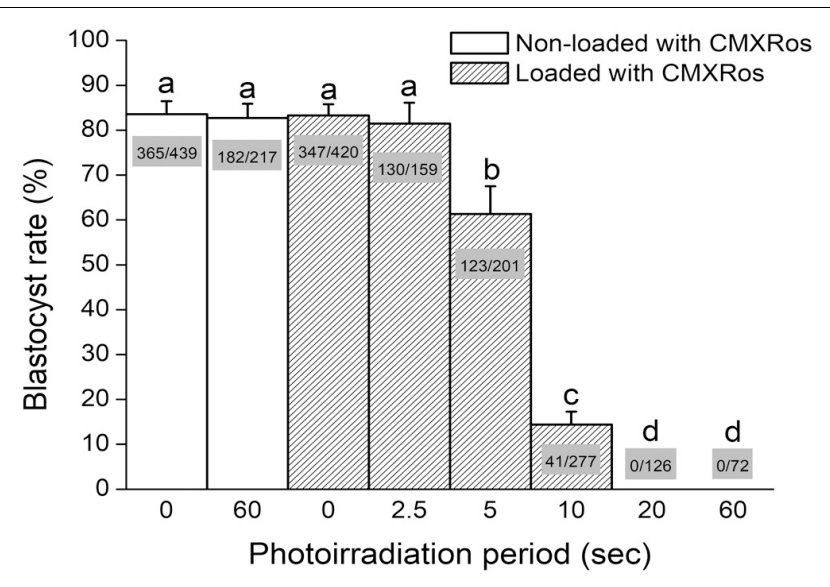

FIGURE 1 | Photoirradiation of CMXRos-loaded zygotes prevents development into blastocysts. Percentage of NZB zygotes that developed into blastocysts after $96 \mathrm{~h}$ of in vitro culture. Zygotes were either loaded or not with CMXRos prior to photoirradiation. Bar insets represent the number of blastocysts in relation to total number of cultured zygotes. Different letters over bars depict statistical difference $(P<0.05)$.

10, 20, or $60 \mathrm{~s}$ before in vitro culture and analysis of blastocyst development. Zygotes photoirradiated for either 0 or $60 \mathrm{~s}$, without prior incubation with CMXRos, were used as controls. As a result, photoirradiation of non-loaded zygotes for $60 \mathrm{~s}$ did not impact blastocyst rate in comparison with zygotes that were neither incubated with CMXRos nor photoirradiated (Figure 1). Likewise, no effect was seen when CMXRos-loaded zygotes were photoirradiated for 0 or $2.5 \mathrm{~s}$ (Figure 1). Yet, photoirradiation for $5 \mathrm{~s}$ or more progressively impacted on blastocyst rate $(P<0.05)$; a photoirradiation period of 20 or $60 \mathrm{~s}$ was sufficient to completely prevent blastocyst formation (Figure 1). In summary, these findings show a linear impact of photoirradiation time on blastocyst rate, which relied on the prior loading with CMXRos.

\section{Photoirradiated Mitochondria Injected Into Zygotes Are Selected Against During Early Embryogenesis}

To investigate whether damaged mitochondria are selectively eliminated during early embryogenesis, donor zygotes (containing NZB mtDNA) were loaded with CMXRos and photoirradiated for either 0 or $20 \mathrm{~s}$. We chose a 20-s exposure time given this was the shortest period that completely precluded development into blastocysts (Figure 1). To validate our system, we first assessed in recipient zygotes (CT-P0 and CT-P20) the levels of NZB mtDNA following CT. As a result, comparable levels $(P>0.05)$ of NZB mtDNA were present in CT-P0 (30.8 \pm 1.73$)$ and CT-P20 (30.6 \pm 1.73$)$ zygotes (Figure 2A). Similarly, mtDNA copy number (sum of B6 and NZB mtDNA) was not different $(P>0.05)$ between CT-P0 $(365,022 \pm 33,062)$ and CT-P20 $(365,704 \pm 33,314)$ zygotes (Figure 2B). These zygotes also presented similar mtDNA copy number $(P>0.05)$ compared with zygotes not subjected to CT: B6-control (348,850 $\pm 23,696)$, NZB-P0 (375,461 $\pm 33,388)$, and NZB-P20 $(359,852 \pm 23,132)$. In summary, neither photoirradiation nor CT altered the levels of NZB and total mtDNA in pronuclear zygotes.

We next sought to assess the levels of NZB mtDNA after development of CT-derived zygotes into blastocysts. Toward this, CT-P0 and CT-P20 embryos were cultured in vitro for $96 \mathrm{~h}$, reaching the blastocyst stage with similar rates (CT-P0 $=92.3 \%$ vs. CT-P20 $=83.8 \%)$ when compared to that of B6-control $(84.5 \%)$ and NZB-P0 (77.9\%) embryos. In comparison, only $5.8 \%$ of NZBP20 zygotes developed into blastocysts $(P<0.05)$. In regard of the levels of CT-derived mitochondria, similar $(P>0.05)$ levels of NZB mtDNA were found between blastocysts $(31.4 \% \pm 1.43)$ and zygotes $(30.8 \% \pm 1.73)$ of the CT-P0 group (Figure 2A). Conversely, the levels of NZB mtDNA in CT-P20 embryos dropped $(P=0.008)$ from $30.6 \% \pm 1.73$ in zygotes to $24.7 \% \pm 1.43$ in blastocysts (Figure 2A). The levels of NZB mtDNA also proved to be lower $(P<0.05)$ in CT-P20 than CT-P0 blastocysts (Figure 2A). On the other hand, mtDNA copy number remained stable (Figure 2B) between groups at the blastocyst stage (CT$\mathrm{P} 0=336,497 \pm 14,551$ vs. CT-P20 $=371,063 \pm 20,054)$. This was also true when compared with NZB-P0 $(352,179 \pm 15,704)$ and B6-control $(366,065 \pm 11,322)$ blastocysts. Therefore, these findings provide evidence that photoirradiated mitochondria introduced into zygotes were eliminated during development into blastocysts.

\section{Rapamycin Treatment Precludes Elimination of Photoirradiated Mitochondria During Early Embryogenesis}

To investigate whether autophagy was linked with the drop in the levels of photoirradiated mitochondria in blastocysts, zygotes subjected to CT were cultured in the presence of rapamycin-an autophagy agonist (Lee et al., 2011; Gilkerson et al., 2012; Dai et al., 2014). As a result, the rapamycin treatment precluded elimination of photoirradiated-derived mtDNA, resulting in similar $(P>0.05)$ levels of NZB and total mtDNA between CT-P0 and CT-P20 blastocysts (Figures 3A,B). Additionally, analysis of CT-derived embryos (at the twocell stage) provided no evidence of increased mitochondriaautophagosome colocalization, regardless of the rapamycin treatment (Figure 4). Taken together, these results do not support a link between autophagy and elimination of photoirradiatedderived mitochondria during early embryogenesis.

\section{DISCUSSION}

Our present findings provide new evidence that damaged mitochondria are eliminated during early embryogenesis through an autophagy-independent mechanism.

After absorption of light, certain biocompatible photosensitizers are capable of generating reactive species (i.e., hydroxyl radicals and singlet oxygen), which may damage neighboring biomolecules such as membrane unsaturated lipids, proteins, and DNAs (Foote, 1968). Given that some photosensitizers accumulate in specific 
A

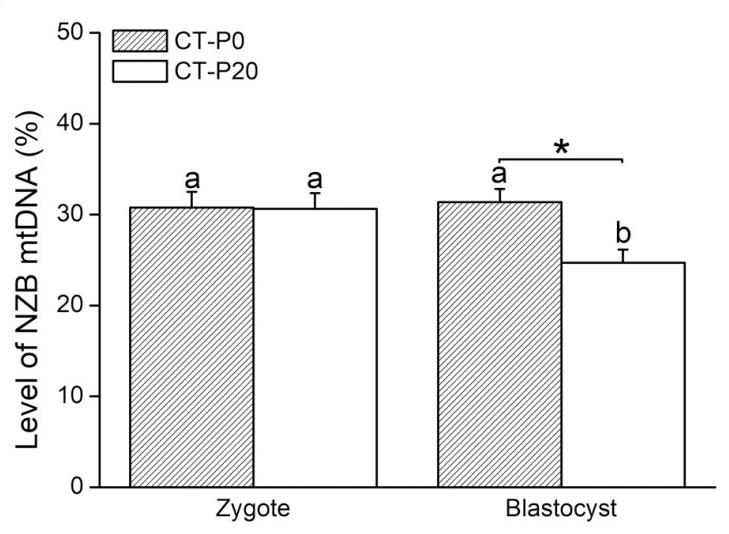

B

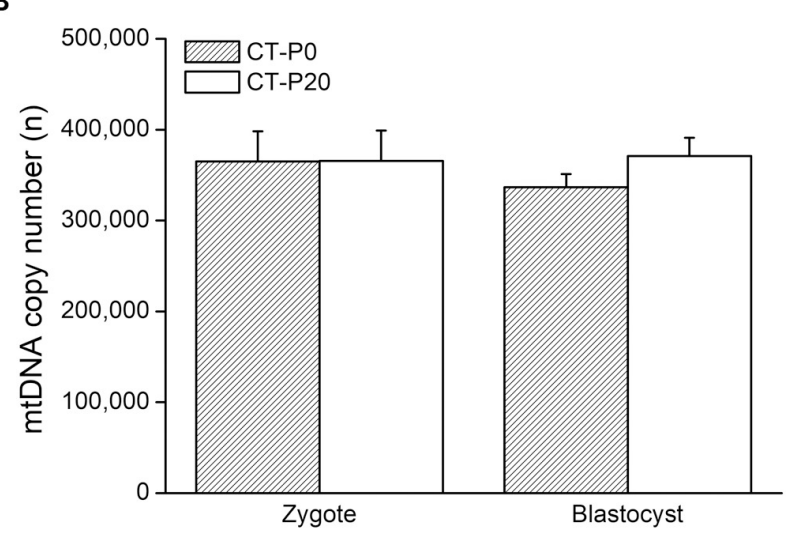

FIGURE 2 | Photoirradiated mitochondria injected into zygotes are selected against during early embryogenesis. CMXRos-loaded zygotes containing mtDNA of NZB origin were photoirradiated for either 0 (P0) or 20 (P20) s to induce mitochondrial damage. Mitochondria from these zygotes were injected by cytoplasmic transfer (CT) into B6 zygotes, resulting in CT-P0 and CT-P20 groups, respectively. CT-derived embryos were assessed at zygote and blastocyst stage as for the levels of NZB (A) and total (B) mtDNA. Different letters over bars depict statistical difference within group $(P<0.05)$. * Statistical difference within stage $(P=0.008)$.

A

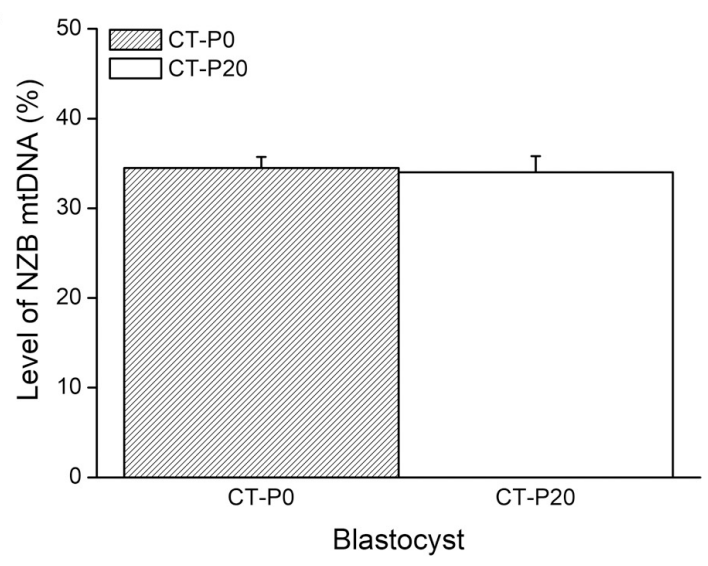

B

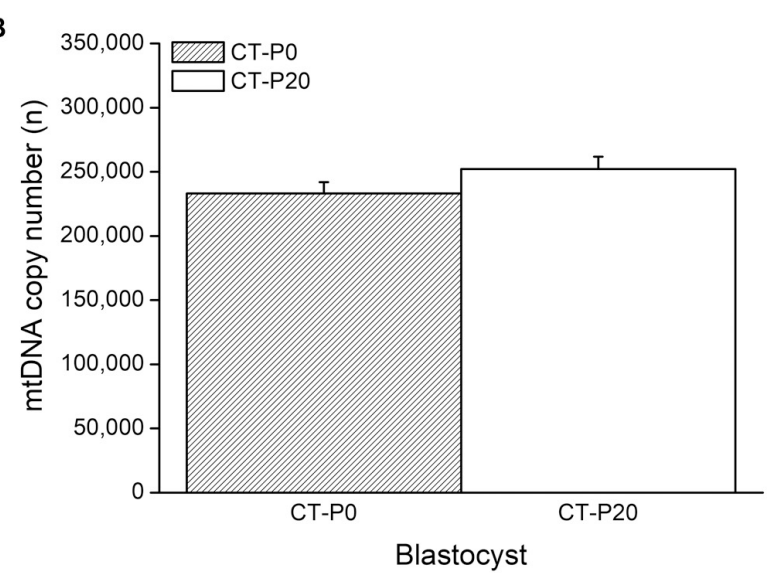

FIGURE 3 | Rapamycin treatment precludes elimination of photoirradiated mitochondria during early embryogenesis. CMXRos-loaded zygotes containing mtDNA of NZB origin were photoirradiated for either 0 (P0) or 20 (P20) s to induce mitochondrial damage. Mitochondria from these zygotes were injected by cytoplasmic transfer (CT) into B6 zygotes, resulting in CT-P0 and CT-P20 groups, respectively. CT-derived blastocysts were assessed as for the levels of NZB (A) and total (B) mtDNA. No statistical difference $(P>0.05)$.

subcellular compartments, damage can be efficiently targeted to mitochondria (Gabrielli et al., 2004; Oliveira et al., 2011; Hammerer et al., 2018; Taba et al., 2018). In this respect, CMXRos has been shown, both in cultured cells and mouse oocytes, to be a potent mitochondrial photosensitizer (Minamikawa et al., 1999; Lum et al., 2002; Palermo et al., 2002; Lum and Nagley, 2003; Takeuchi et al., 2005). Hence, we have used CMXRos and photoirradiation to specifically induce mitochondrial damage in mouse zygotes. As a result, we found that either CMXRos or photoirradiation alone have no effect on development into blastocysts. Yet, photoirradiation of CMXRos-loaded zygotes for $5 \mathrm{~s}$ or more led to a linear decline (up to $20 \mathrm{~s}$ ) on blastocyst rate. These results corroborate previous findings that photosensitization of oocytes leads to mitochondrial dysfunction and developmental arrest after fertilization (Palermo et al., 2002; Thouas et al., 2004, 2006; Takeuchi et al., 2005).
Given the time-depend effect of photoirradiation, we decided to photoirradiate NZB zygotes, loaded with CMXRos, for either 0 or $20 \mathrm{~s}$. Next, their cytoplasm was transferred into B6 zygotes aiming to track injected mitochondria in blastocysts. Injected and recipient mitochondria were distinguished based on mtDNA origin, respectively, NZB and B6. Importantly, $\sim 30 \%$ of NZB mtDNA were present in zygotes, regardless of the photoirradiation treatment. However, the levels of NZB mtDNA dropped in blastocysts only when photoirradiated-zygotes were used as cytoplasmic donors. Once zygotes injected with either photoirradiated or nonphotoirradiated cytoplasm developed into blastocysts with similar rates, this drop cannot be attributed to an impact of CT on embryogenesis. Moreover, mtDNA copy number remained stable between stages (zygotes vs. blastocysts) and treatments (photoirradiated vs. non-photoirradiated), indicating 


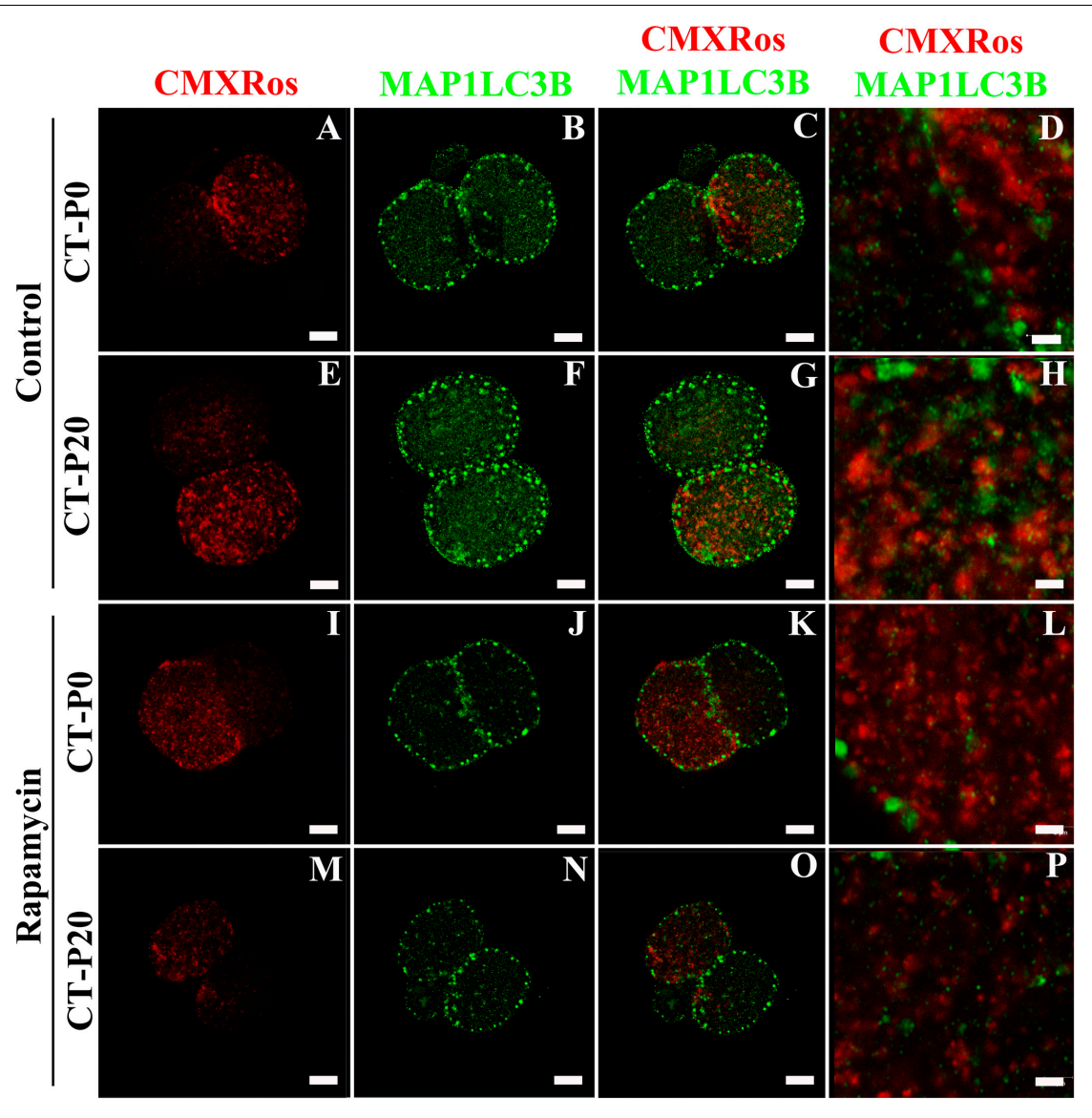

FIGURE 4 | Autophagy is not linked with elimination of photoirradiated mitochondria in early embryos. CMXRos-loaded zygotes were photoirradiated for either 0 (PO) or 20 (P20) s to induce mitochondrial damage. Mitochondria from these zygotes were injected by cytoplasmic transfer (CT) into recipient zygotes, resulting in CT-P0 (A-D and I-L) and CT-P20 (E-H and M-P) groups, respectively. CT-derived zygotes were cultured in vitro for $24 \mathrm{~h}$ in either absence (A-H) or presence (I-P) of rapamycin. Autophagosomes in two-cell embryos were detected by immunofluorescence using a primary antibody against MAP1LC3B (green; B,F,J,N). Injected mitochondria were visualized based on CMXRos fluorescence (red; A,E,I,M). Pictures were merged to assess mitochondria-autophagosome colocalization $\mathbf{( C , G , K , O , D , H , L , P ) . ~ B a r s ~ i n ~ ( A - C , E - G , I - K , M - O ) , ~ a n d ~} \mathbf{M}-\mathbf{O}$ correspond to $10 \mu \mathrm{m}$, while in (D,H,L,P), they correspond to $2 \mu \mathrm{m}$.

that photoirradiated-derived mtDNA was replaced by recipient mtDNA in blastocysts.

There is mounting evidence in support of purifying selection acting in the female germline to prevent the accumulation of deleterious mtDNA mutations (Sato et al., 2007; Fan et al., 2008; Stewart et al., 2008; Freyer et al., 2012; Sharpley et al., 2012; Li et al., 2016; Floros et al., 2018; Lieber et al., 2019; Wei et al., 2019). Among other stages of germline development, purifying selection may take place during early embryogenesis as reported by Lee et al. (2012) and LatorrePellicer et al. (2019). In agreement with these reports, our present data indicate that the levels of NZB mtDNA dropped in blastocysts only when it derived from photoirradiated cytoplasm. Given that photoirradiation leads to mitochondrial damage (Minamikawa et al., 1999; Lum et al., 2002; Palermo et al., 2002; Lum and Nagley, 2003; Takeuchi et al., 2005), including on mtDNA (Battogtokh et al., 2018), we argue that photoirradiated mitochondria were targeted for destruction in preimplantation embryos (Wang et al., 2012). This hypothesis is in keeping with autophagic elimination of paternal mitochondria following fertilization (Rojansky et al., 2016), suggesting that a similar mechanism might be involved with elimination of dysfunctional mitochondria inherited from the oocyte.

To address the hypothesis that photoirradiated mitochondria were destroyed by autophagy, injected embryos were cultured in the presence of rapamycin. We expected with this treatment to enhance the drop in the levels of NZB mtDNA as rapamycin is a canonical inducer of macroautophagy; by inhibiting mTORC1, rapamycin induces autophagosome formation and degradation of cellular components such as dysfunctional mitochondria (Kim et al., 2002; Narendra et al., 2008; Twig et al., 2008; Suen et al., 2010; Gilkerson et al., 2012; Dai et al., 2014). In opposite to our prediction, rapamycin prevented the drop in NZB mtDNA associated with injection of photoirradiated cytoplasm. Although difficult to explain, we propose that rapamycin mitigated mitochondrial damage induced by photoirradiation. This hypothesis is supported by a previous report showing that rapamycin upregulates DNA repair enzyme OGG1 (Habib et al., 2010). Thus, rapamycin might have countered mitochondrial damage by enhancing mtDNA repair on photoirradiated-derived 
mitochondria. In addition, embryos were assessed as for colocation between injected mitochondria and autophagosomes. Two-cell embryos were used as an autophagic wave takes place at this stage (Tsukamoto et al., 2008), coinciding with destruction of paternal mitochondria in mice (Rojansky et al., 2016). However, no skewed colocalization of photoirradiated mitochondria and autophagosomes was seen, even when considering the rapamycin treatment. Together, these data do not implicate autophagy in the elimination of photoirradiated-derived mitochondria.

Our current findings support the hypothesis that damaged mitochondria are destroyed during early embryogenesis, suggesting that the same mechanism might take place to counter expansion of deleterious mtDNA mutations. Such mechanism is in accordance with the "Muller's ratchet" theory, which proposes that uniparental inheritance of mtDNA in the absence of recombination would lead to accumulation and fixation of deleterious mutations (Muller, 1964). In fact, few highly deleterious mutations in mtDNA have become fixed in the human population, lending further support to purifying selection (Rand and Kann, 1996; Elson et al., 2004; Rand, 2008; Wei et al., 2019). Considering that deleterious mtDNA mutations may impact mitochondrial function, mutations might be selected against at the organelle level (Burr et al., 2018). In support of this notion, previous reports have provided evidence that autophagy acts to eliminate dysfunctional mitochondria with deleterious mtDNA mutations (Narendra et al., 2008; Twig et al., 2008; Suen et al., 2010; Gilkerson et al., 2012; Dai et al., 2014). Although our findings do not support a link between autophagy and the lower levels of photoirradiated-derived mitochondria in blastocysts, this requires further investigation as it might be a rapamycinindependent mechanism (Yamamoto et al., 2014) or take place at a different embryonic stage (Tsukamoto et al., 2008).

\section{CONCLUSION}

The preimplantation embryo is competent to mitigate the levels of damaged mitochondria. This finding is of relevance for the transmission of mitochondrial disease as a similar mechanism might take place during early embryogenesis to counter expansion of deleterious mtDNA mutations. Limitation of the study: lack of mtDNA sequencing data. Further studies are needed to show whether the lower levels of

\section{REFERENCES}

Battogtokh, G., Choi, Y. S., Kang, D. S., Park, S. J., Shim, M. S., Huh, K. M., et al. (2018). Mitochondria-targeting drug conjugates for cytotoxic, anti-oxidizing and sensing purposes: current strategies and future perspectives. Acta Pharm. Sin. B 8, 862-880. doi: 10.1016/j.apsb.2018.05.006

Burr, S. P., Pezet, M., and Chinnery, P. F. (2018). Mitochondrial DNA heteroplasmy and purifying selection in the mammalian female germ line. Dev. Growth Diff. 60, 21-32. doi: 10.1111/dgd.12420

Chiaratti, M. R., Bressan, F. F., Ferreira, C. R., Caetano, A. R., Smith, L. C., Vercesi, A. E., et al. (2010). Embryo mitochondrial DNA depletion is reversed during early embryogenesis in cattle. Biol. Reprod. 82, 76-85. doi: 10.1095/biolreprod. 109.077776

Cree, L. M., Samuels, D. C., de Sousa Lopes, S. C., Rajasimha, H. K., Wonnapinij, P., Mann, J. R., et al. (2008). A reduction of mitochondrial DNA molecules damaged mitochondria in blastocysts are linked with elimination of potentially deleterious mtDNA mutations derived from photoirradiation.

\section{DATA AVAILABILITY STATEMENT}

The datasets generated for this study are available on request to the corresponding author.

\section{ETHICS STATEMENT}

The animal study was reviewed and approved by the Animal Care and Use Committee at Universidade de São Paulo (USPprotocol number 13.1.1832.74.8).

\section{AUTHOR CONTRIBUTIONS}

MRC designed the experiments and wrote the manuscript. TM, CM, MDC, and MRC carried out the experiments, data organization, and statistical analyses. FG and FM contributed new reagents and analytical tools. All authors read and approved the final manuscript.

\section{FUNDING}

This work was funded by the Fundação de Amparo à Pesquisa do Estado de São Paulo (FAPESP/Brazil-grants \# 2009/540354, 2012/50231-6, 2012/12951-7, 2013/13869-5, 2017/04372-0, and 2017/05899-2), the Conselho Nacional de Desenvolvimento Científico e Tecnológico (CNPq/Brazil—grant \# 472507/2012-5), and the Coordenação de Aperfeiçoamento de Pessoal de Nível Superior-Brazil (CAPES)_Finance Code 001.

\section{ACKNOWLEDGMENTS}

We deeply thank Dr. Nadja C. Souza-Pinto (IQ/USP) and Dr. Juliano R. Sangalli (FZEA/USP) for invaluable suggestions to this work, and Thiago B. Rodrigues for technical support.

during embryogenesis explains the rapid segregation of genotypes. Nat. Genet. 40, 249-254. doi: 10.1038/ng.2007.63

Dai, Y., Zheng, K., Clark, J., Swerdlow, R. H., Pulst, S. M., Sutton, J. P., et al. (2014). Rapamycin drives selection against a pathogenic heteroplasmic mitochondrial DNA mutation. Hum. Mol. Genet. 23, 637-647. doi: 10.1093/hmg/ddt450

Elson, J. L., Turnbull, D. M., and Howell, N. (2004). Comparative genomics and the evolution of human mitochondrial DNA: assessing the effects of selection. Am. J. Hum. Genet. 74, 229-238. doi: 10.1086/381505

Erbach, G. T., Lawitts, J. A., Papaioannou, V. E., and Biggers, J. D. (1994). Differential growth of the mouse preimplantation embryo in chemically defined media. Biol. Reprod. 50, 1027-1033. doi: 10.1095/biolreprod50.5.1027

Fan, W., Waymire, K. G., Narula, N., Li, P., Rocher, C., Coskun, P. E., et al. (2008). A mouse model of mitochondrial disease reveals germline selection against severe mtDNA mutations. Science 319, 958-962. doi: 10.1126/science.114 7786 
Floros, V. I., Pyle, A., Dietmann, S., Wei, W., Tang, W. C. W., Irie, N., et al. (2018). Segregation of mitochondrial DNA heteroplasmy through a developmental genetic bottleneck in human embryos. Nat. Cell. Biol. 20, 144-151. doi: 10.1038/ s41556-017-0017-8

Foote, C. S. (1968). Mechanisms of photosensitized oxidation. Science 162, 963970. doi: 10.1126/science.162.3857.963

Freyer, C., Cree, L. M., Mourier, A., Stewart, J. B., Koolmeister, C., Milenkovic, D., et al. (2012). Variation in germline mtDNA heteroplasmy is determined prenatally but modified during subsequent transmission. Nat. Genet. 44, 12821285. doi: 10.1038/ng.2427

Gabrielli, D., Belisle, E., Severino, D., Kowaltowski, A. J., and Baptista, M. S. (2004). Binding, aggregation and photochemical properties of methylene blue in mitochondrial suspensions. Photochem. Photobiol. 79:227. doi: 10.1562/be03-27.1

Gilkerson, R. W., De Vries, R. L. A., Lebot, P., Wikstrom, J. D., Torgyekes, E., Shirihai, O. S., et al. (2012). Mitochondrial autophagy in cells with mtDNA mutations results from synergistic loss of transmembrane potential and mTORC1 inhibition. Hum. Mol. Genet. 21, 978-990. doi: 10.1093/hmg/ddr529

Habib, S. L., Kasinath, B. S., Arya, R. R., Vexler, S., and Velagapudi, C. (2010). Novel mechanism of reducing tumourigenesis: upregulation of the DNA repair enzyme OGG1 by rapamycin-mediated AMPK activation and mTOR inhibition. Eur. J. Cancer 46, 2806-2820. doi: 10.1016/j.ejca.2010.06.117

Hammerer, F., Poyer, F., Fourmois, L., Chen, S., Garcia, G., Teulade-Fichou, M. P., et al. (2018). Mitochondria-targeted cationic porphyrin-triphenylamine hybrids for enhanced two-photon photodynamic therapy. Bioorg. Med. Chem. 26, 107-118. doi: 10.1016/j.bmc.2017.11.024

Jenuth, J., Peterson, A., Fu, K., and Shoubridge, E. (1996). Random genetic drift in the female germline explains the rapid segregation of mammalian mitochondrial DNA. Nat. Genet. 14, 146-151. doi: 10.1038/ng1096-146

Kim, D. H., Sarbassov, D. D., Ali, S. M., King, J. E., Latek, R. R., ErdjumentBromage, H., et al. (2002). mTOR interacts with raptor to form a nutrientsensitive complex that signals to the cell growth machinery. Cell 110, 163-175. doi: 10.1016/S0092-8674(02)00808-5

Latorre-Pellicer, A., Lechuga-Vieco, A. V., Johnston, I. G., Hämäläinen, R. H., Pellico, J., Justo-Méndez, R., et al. (2019). Regulation of mother-to-offspring transmission of mtDNA heteroplasmy. Cell Metab. 30, 1120.e5-1130.e5. doi: 10.1016/j.cmet.2019.09.007

Lee, H., Ma, H., Juanes, R., Tachibana, M., Sparman, M., Woodward, J., et al. (2012). Rapid mitochondrial DNA segregation in primate preimplantation embryos precedes somatic and germline bottleneck. Cell. Rep. 1, 506-515. doi: 10.1016/j. celrep.2012.03.011.Rapid

Lee, S. E., Hwang, K. C., Sun, S. C., Xu, Y. N., and Kim, N. H. (2011). Modulation of autophagy influences development and apoptosis in mouse embryos developing in vitro. Mol. Reprod. Dev. 78, 498-509. doi: 10.1002/mrd.21331

Li, M., Rothwell, R., Vermaat, M., Wachsmuth, M., Schröder, R., Laros, J. F. J., et al. (2016). Transmission of human mtDNA heteroplasmy in the Genome of the Netherlands families: support for a variable-size bottleneck. Genome Res. 26, 417-426. doi: 10.1101/gr.203216.115

Lieber, T., Jeedigunta, S. P., Palozzi, J. M., Lehmann, R., and Hurd, T. R. (2019). Mitochondrial fragmentation drives selective removal of deleterious mtDNA in the germline. Nature 570, 380-384. doi: 10.1038/s41586-019-1213-4

Lum, M.-G., Minamikawa, T., and Nagley, P. (2002). Microscopic photosensitization: a new tool to investigate the role of mitochondria in cell death. Sci. World J. 2, 1198-1208. doi: 10.1100/tsw.2002.227

Lum, M.-G., and Nagley, P. (2003). Two phases of signalling between mitochondria during apoptosis leading to early depolarisation and delayed cytochrome $\mathrm{c}$ release. J. Cell Sci. 116, 1437-1447. doi: 10.1242/jcs.00320

Luo, S., Valencia, C. A., Zhang, J., Lee, N. C., Slone, J., Gui, B., et al. (2018). Biparental inheritance of mitochondrial DNA in humans. Proc. Natl. Acad. Sci. U.S.A. 115, 13039-13044. doi: 10.1073/pnas.1810946115

Machado, T. S., Macabelli, C. H., Sangalli, J. R., Rodrigues, T. B., Smith, L. C., Meirelles, F. V., et al. (2015). Real-Time PCR quantification of heteroplasmy in a mouse model with mitochondrial DNA of C57BL/6 and NZB/BINJ strains. PLoS One 10:e0133650. doi: 10.1371/journal.pone.0133650

Minamikawa, T., Sriratana, A., Williams, D. A., Bowser, D. N., Hill, J. S., and Nagley, P. (1999). Chloromethyl-X-rosamine (MitoTracker Red) photosensitises mitochondria and induces apoptosis in intact human cells. J. Cell Sci. 112(Pt 1), 2419-2430.
Muller, H. J. (1964). The relation of recombination to mutational advance. Mut. Res. 1, 2-9. doi: 10.1016/0027-5107(64)90047-8

Nagy, A., Gertsenstein, M., Vintersten, K., and Behringer, R. (2003). Manipulating the Mouse Embryo: A Laboratory Manual, 3rd Edn. New York, NY: Cold Spring Harbor Laboratory Press.

Narendra, D., Tanaka, A., Suen, D.-F., and Youle, R. J. (2008). Parkin is recruited selectively to impaired mitochondria and promotes their autophagy. J. Cell. Biol. 183, 795-803. doi: 10.1083/jcb.200809125

Oliveira, C. S., Turchiello, R., Kowaltowski, A. J., Indig, G. L., and Baptista, M. S. (2011). Major determinants of photoinduced cell death: subcellular localization versus photosensitization efficiency. Free Radic. Biol. Med. 51, 824-833. doi: 10.1016/j.freeradbiomed.2011.05.023

Palermo, G. D., Takeuchi, T., and Rosenwaks, Z. (2002). Technical approaches to correction of oocyte aneuploidy. Hum. Reprod. 17, 2165-2173. doi: 10.1093/ humrep/17.8.2165

Rand, D. M. (2008). Mitigating mutational meltdown in mammalian mitochondria. PLoS Biol. 6:e35. doi: 10.1371/journal.pbio.0060035

Rand, D. M., and Kann, L. M. (1996). Excess amino acid polymorphism in mitochondrial DNA: contrasts among genes from Drosophila, mice, and humans. Mol. Biol. Evol. 13, 735-748. doi: 10.1093/oxfordjournals.molbev. a025634

Rojansky, R., Cha, M. Y., and Chan, D. C. (2016). Elimination of paternal mitochondria in mouse embryos occurs through autophagic degradation dependent on PARKIN and MUL1. eLife 5, 1-18. doi: 10.7554/eLife.17896

Sato, A., Nakada, K., Shitara, H., Kasahara, A., Yonekawa, H., and Hayashi, J.-I. (2007). Deletion-mutant mtDNA increases in somatic tissues but decreases in female germ cells with age. Genetics 177, 2031-2037. doi: 10.1534/genetics.107. 081026

Schon, E. A., DiMauro, S., and Hirano, M. (2012). Human mitochondrial DNA: roles of inherited and somatic mutations. Nat. Rev. Genet. 13, 878-890. doi: $10.1038 / \operatorname{nrg} 3275$

Sharpley, M. S., Marciniak, C., Eckel-Mahan, K., McManus, M., Crimi, M., Waymire, K., et al. (2012). Heteroplasmy of mouse mtDNA is genetically unstable and results in altered behavior and cognition. Cell 151, 333-343. doi: 10.1016/j.cell.2012.09.004

Stewart, J. B., and Chinnery, P. F. (2015). The dynamics of mitochondrial DNA heteroplasmy: implications for human health and disease. Nat. Rev. Genet. 16, 530-542. doi: 10.1038/nrg3966

Stewart, J. B., Freyer, C., Elson, J. L., Wredenberg, A., Cansu, Z., Trifunovic, A., et al. (2008). Strong purifying selection in transmission of mammalian mitochondrial DNA. PLoS Biol. 6:e10. doi: 10.1371/journal.pbio.0060010

Suen, D.-F., Narendra, D. P., Tanaka, A., Manfredi, G., and Youle, R. J. (2010). Parkin overexpression selects against a deleterious mtDNA mutation in heteroplasmic cybrid cells. Proc. Natl. Acad. Sci. U.S.A. 107, 11835-11840. doi: 10.1073/pnas.0914569107

Taba, F., Onoda, A., Hasegawa, U., Enoki, T., Ooyama, Y., Ohshita, J., et al. (2018). Mitochondria-targeting polyamine-protoporphyrin conjugates for photodynamic therapy. ChemMedChem 13, 15-19. doi: $10.1002 / \mathrm{cmdc}$. 201700467

Takeuchi, T., Neri, Q. V., Katagiri, Y., Rosenwaks, Z., and Palermo, G. D. (2005). Effect of treating induced mitochondrial damage on embryonic development and epigenesis. Biol. Reprod. 72, 584-592. doi: 10.1095/biolreprod.104.032391

Thouas, G. A., Trounson, A. O., and Jones, G. M. (2006). Developmental effects of sublethal mitochondrial injury in mouse oocytes. Biol. Reprod. 74, 969-977. doi: 10.1095/biolreprod.105.048611

Thouas, G. A., Trounson, A. O., Wolvetang, E. J., and Jones, G. M. (2004). Mitochondrial dysfunction in mouse oocytes results in preimplantation embryo arrest in vitro. Biol. Reprod. 71, 1936-1942. doi: 10.1095/biolreprod.104.033589

Tsukamoto, S., Kuma, A., Murakami, M., Kishi, C., Yamamoto, A., and Mizushima, N. (2008). Autophagy is essential for preimplantation development of mouse embryos. Science 321, 117-120. doi: 10.1126/science.1154822

Twig, G., Elorza, A., Molina, A. J. A., Mohamed, H., Wikstrom, J. D., Walzer, G., et al. (2008). Fission and selective fusion govern mitochondrial segregation and elimination by autophagy. EMBO J. 27, 433-446. doi: 10.1038/sj.emboj. 7601963

Wai, T., Teoli, D., and Shoubridge, E. A. (2008). The mitochondrial DNA genetic bottleneck results from replication of a subpopulation of genomes. Nat. Genet. 40, 1484-1488. doi: 10.1038/ng.258 
Wallace, D. C., and Chalkia, D. (2013). Mitochondrial DNA genetics and the heteroplasmy conundrum in evolution and disease. Cold Spring Harb. Perspect. Biol. 5:a021220. doi: 10.1101/cshperspect.a02 1220

Wang, Y., Nartiss, Y., Steipe, B., McQuibban, G. A., and Kim, P. K. (2012). ROSinduced mitochondrial depolarization initiates PARK2/PARKIN-dependent mitochondrial degradation by autophagy. Autophagy 8, 1462-1476. doi: 10 . 4161/auto.21211

Wei, W., Pagnamenta, A. T., Gleadall, N., Sanchis-Juan, A., Stephens, J., Broxholme, J., et al. (2020). Nuclear-mitochondrial DNA segments resemble patternally inherited mitochondrial DNA in humans. Nat. Commun. 11:1740. doi: 10.1038/s41467-020-15336-3

Wei, W., Tuna, S., Keogh, M. J., Smith, K. R., Aitman, T. J., Beales, P. L., et al. (2019). Germline selection shapes human mitochondrial DNA diversity. Science 364:eaau6520. doi: 10.1126/science.aau6520
Yamamoto, A., Mizushima, N., and Tsukamoto, S. (2014). Fertilization-induced autophagy in mouse embryos is independent of mTORC1. Biol. Reprod. 91:7. doi: 10.1095/biolreprod.113.115816

Conflict of Interest: The authors declare that the research was conducted in the absence of any commercial or financial relationships that could be construed as a potential conflict of interest.

Copyright (c) 2020 Machado, Macabelli, Collado, Meirelles, Guimarães and Chiaratti. This is an open-access article distributed under the terms of the Creative Commons Attribution License (CC BY). The use, distribution or reproduction in other forums is permitted, provided the original author(s) and the copyright owner(s) are credited and that the original publication in this journal is cited, in accordance with accepted academic practice. No use, distribution or reproduction is permitted which does not comply with these terms. 